\title{
Efficacy of active rapid molecular screening and IPC interventions to reduce carbapenem-resistant Enterobacteriaceae infections in emergency intensive care unit - a prospective, multi-stage study
}

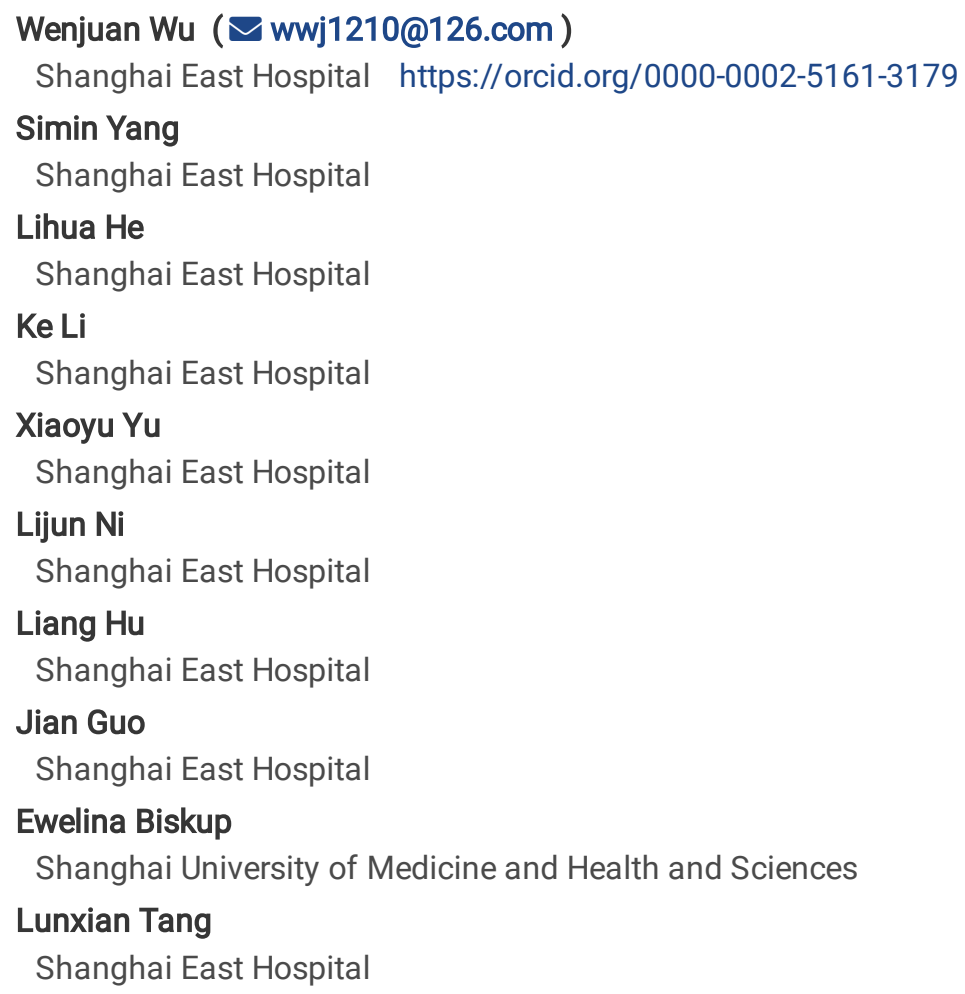




\section{Abstract}

Background: To investigate whether molecular rapid active screening and infection-prevention and contro|『IPCखinterventions can reduce the colonization and infection of carbapenem-resistant Enterobacteriaceae (CRE) in a general emergency intensive care unit (EICU).

Methods: The study was designed as a before-and-after quasi-experiment. It was conducted in 3 stages. During stage 1, April 2018, the environment was prepared and the staff in EICU was trained. Stage 2 was the main experimental stage from May 2018 to January 2019. The active screening was tested by semi-nested real-time fluorescent PCR (polymerase chain reaction) detection with rectal swabs from all the patients on admission to EICU and the results would be feedback in 1 hour and other IPC interventions were conducted in strict supervision in this stage. In the last stage (stage 3), February 2019-April 2019, only active screening was strictly executed. Other IPC interventions were carried out by health workers without supervision. In the meantime, the atient information and culture results from clinical laboratory from January 2017-April 2019 were collected.

Results: In this 1-year study, a total of 217 patients were enrolled. There were $23.04 \%$ of the patients were initially colonized or infected with CRE as tested by active molecular screening. The clinical culture detection rate of CRE colonization/infection was $3.37 \%$ during the baseline stage (before this study was carried out), from January 2017-March 2018. The rate decreased significantly to $1.20 \%(p<0.05)$ during the main experimental period in which active screening and IPC intervention were executed strictly. However, the rate increased again to $6.12 \%$ in stage 3 in which only active screening were carried out in supervision. Based on the clinical characteristics of the EICU patients, we found there's a higher probability that the patients had invasive devices or skin-barrier damage on admission or had antibiotic use before admission were colonized or infected with CRE $(p<0.05)$.

Conclusions : Rapid active screening by molecular detection and other IPC interventions conducted in supervision showed a significant reduction of CRE in nosocomial infection. The key to reduce the spread of CRE in the EICU is that all medical staff and healthcare workers execute IPC interventions strictly.

\section{Introduction}

Carbapenem-resistant Enterobacteriaceae (CRE) represent a serious therapeutic problem due to their pronounced multidrug resistance, with main pathogens being Klebsiella pneumoniae, Escherichia coli, and other Enterobacteriaceae. The worldwide spread of CRE is a global burden and has become one of the priorities of the Centers for Disease Control (CDC) [1]. In the last decades, along with the advent of Extended Spectrum Beta-Lactamase (ESBL) -producing enterobacteria, carbapenems have been used more frequently due to their broad antibacterial spectrum, thus contributed greatly to the drastic raise of the CRE problem. [2]. In Europe and South America, the situation reached alarming levels, causing about 140,000 cases of healthcare-associated infections annually. [3] In some parts of Asia, especially eastern China, CRE have become endemic and reached epidemic dimensions. [4].

Infections with carbapenem-resistant gram-negative bacilli (CR-GNB), such as CRE, carbapenem-resistant Acinetobacter baumannii (CRAB), carbapenem-resistant Pseudomonas aeruginosa (CRPsA), are predominantly associated with high morbidity, mortality, and heavy economic burden [5]. Despite a variety of preventive measures and implemented intervention programs to control nosocomial infections, the culture detection rate of CRE has increased continuously, reaching a total incidence rate in ICU from $2 \%$ to $7 \%$ and a mortality rate of $18 \%$ to $48 \%$, reaching even $78 \%$ in the patients infected with carbapenem-resistant Klebsiella pneumoniae after transplantation in Germany, 2013. [6] [7] [8] [9]. In 2017, a CRE clinical and economics outcomes model was developed in the United States to evaluate the health and financial burden of CRE, reporting a doubling of costs and a 5.1 fold increase of incidence rates, causing higher expenses than many chronic diseases. [10]

Recent studies showed that the majority of CRE infections in China are caused by carbapenem-resistant Klebsiella pneumoniae (CR-KP), mostly leading to serious infection in the ICUs [11]. The major mechanisms of carbapenem-resistance in these strains include production of carbapenemases, production of efflux pumps and porin mutation or loss. [12] From 2010 to 2014, the database showed that the average probability of CRE infection from 15 large teaching hospitals in 13 regions of China increased from 1.9\% to 5\%. [13] The resistance rate of Klebsiella pneumoniae to carbapenems has increased by 10\% from 2005 to 2014. [14]This is the highest numbert among all Gram-negative bacteria [15]. In our institution, the Shanghai East Hospital, the detection rates of CR-KP recorded from 2015 to 2017, ranged between $41.3 \%$ to $45.5 \%$. This value was much higher than the reportd average in Shanghai $(27.3 \%$, data collected by CHINET, China Antimicrobial Surveillance Network) and attributed mainly to CR-KP infections in the ICU ward of our hospital, where the antibiotic resistance rate reached $61.12 \%$ in 2017 . 
The WHO published guidelines for the infection prevention and control (IPC) of CRE, CR-AB and CRPsA, including hand hygiene, patient surveillance, contact precautions, patient isolation (single isolation or concentration), environmental disinfection, environment surveillance, monitoring auditing and feedback [16]. However, the translations of the guidelines into the clinic was rather ineffective in China. One of the reasons was that the available global literature mostly reported active screenings conducted by bacterial culture tests (time factor: at least 24h to feedback the results) and the data were collected in settings that are unusual for China, e.g. ICUs with advanced facilities such as 1 bed-room, isolation rooms etc. [17] In addition, in real-world, the IPC interventions implemented in China mostly covered hand hygiene and contact precautions, while infected or colonized patients are under isolation according to local conditions. [18] These are mostly forcing the hospitals to use curtains, one-time hygiene materials and daily utensils, and special signs for isolation were used and contact precautions were strictly applied when private rooms for isolation was not enough. Household items were also separately used and disposed when there were more than one positive patients in the ward.

We conducted a prospective, multi-stages long-term study in order to investigate whether a combination of measures can provide an effective and efficient, as well as persevering results. We aimed to establish whether infection-prevention and control खIPC\together with detection and monitoring of CRE colonizations/infections (active screen at admission) of EICU patients by a rapid, semi-nested real-time fluorescent PCR (polymerase chain reaction) are effective towards the reduction of infection number, mortality and morbidity. At the same time, we provided effective clinical research data to develop China-specific multi-drug resistant prevention and control guidelines.

\section{Material And Methods}

\section{Settings}

The study was conducted at the University Hospital of Shanghai East Hospital, Tongji University School of Medicine, Shanghai. Patients were prospectively recruited from May 2018 through April 2019 in the EICU department, which treats approximately 200-280 critically ill patients annually. The ward consists of 8 beds, including 1 private room and 2 beds in a relatively isolated area.

\section{Infection-control interventions and data collection}

Initially, routine culture data were collected from January 2017 to March 2018. This time period was called the baseline period. In this period, hardly any isolation measures or patient surveillance were done for the patients with CRE. Most of the nurses and doctors did hand hygiene and contact precautions strictly. However, the interventions including hand hygiene, contact precautions and environmental disinfection conducted by the health care workers didn't meet the standard according to our investigation. Clinical specimens were collected for culture only from the patients with infectious clinical symptoms. The data was used as baseline for comparisons to those from stage 3. The study was performed in three stages. In stage 1 (April 2018-May 2018), practical and theoretical EICU staff training on IPC model plan including: hand hygiene, patient surveillance, contact precautions, patient isolation (single isolation or concentration), environmental disinfection, environment surveillance, monitoring auditing and feedback, as well as of the goals, objectives, significance and exact methodology of the project. The whole ward was emptied and the health care workers disinfected the environment with sodium hypochlorite disinfectant, ultraviolet-light radiation and Clinell Universal Wipes which is a combination of benzalkonium chloride, didecyl dimethyl ammonium chloride and polyhexamethylene biguanide (PHMB). Such process of disinfection was also conducted in the area around the bed when a patient left the ward. Hygiene control group examined and confirmed that the ward has been contamination-free prior to the study onset. An additional hand hygiene inspection for all workers was conducted, followed by an intensive training of the study guidelines for sampling and transportation [19]. There isolation guidelines were constructed according to the spatial conditions of the ward in which there are 1 isolation room and 2 semi-isolated rooms( 2 beds in one room and 6 beds in another room with bedside screen and curtain respectively). In case of multiple patients with infectious diseases, bedside screens, separations via curtains and a respective signalization for isolation, with instructions and precautions (clothing, hand disinfection etc.) were placed. Therapeutic supplies and household items were used separately for each patient and disposed after use. Daily operations of the health care workers were strictly directed and supervised, as were the measures of daily disinfection and final disinfection after patients' discharging from the ward [20].

Stage 2 was the main experimental stage, lasting from May 2018 to January 2019. Rrapid active screening by molecular detection and IPC intervention were conducted. At admission to the EICU, rectal swab sampling was performed by a trained nurse and immediately securely transported to the department of clinical laboratory. Each sample was labelled with a Case Report Form (CRF) containing clinical characteristics of the patients, including name, sex, age, admission time, temperature, diagnosis, invasive devices and/or skinbarrier damages, laboratory test results etc. (Table 1) The sample then underwent testing with Gene Xpert, a semi-nested real-time fluorescent PCR method, by a trained lab technician. The Gene Xpert results were returned within one hour(the detection and results 
feedback were done by emergency lab technician during the nights and at weekends), while a culture sampling was set at the same time. During the hospitalization, various type of specimen from the patients (the patients with CRE at admission or clinical symptoms of infection) were sent to laboratory for culture like how was done in baseline period. In the 8 months period of Stage 2, environmental checks and disinfection measures were performed at least twice a day and the environment surveillance twice a month. In addition, sink monitoring and dust avoiding in the process of preparing beds were implemented [21]. The patients' isolation was arranged as described above, according to the results of Gene Xpert [22]

Table 1 CRE Clinical trial Case Report Form

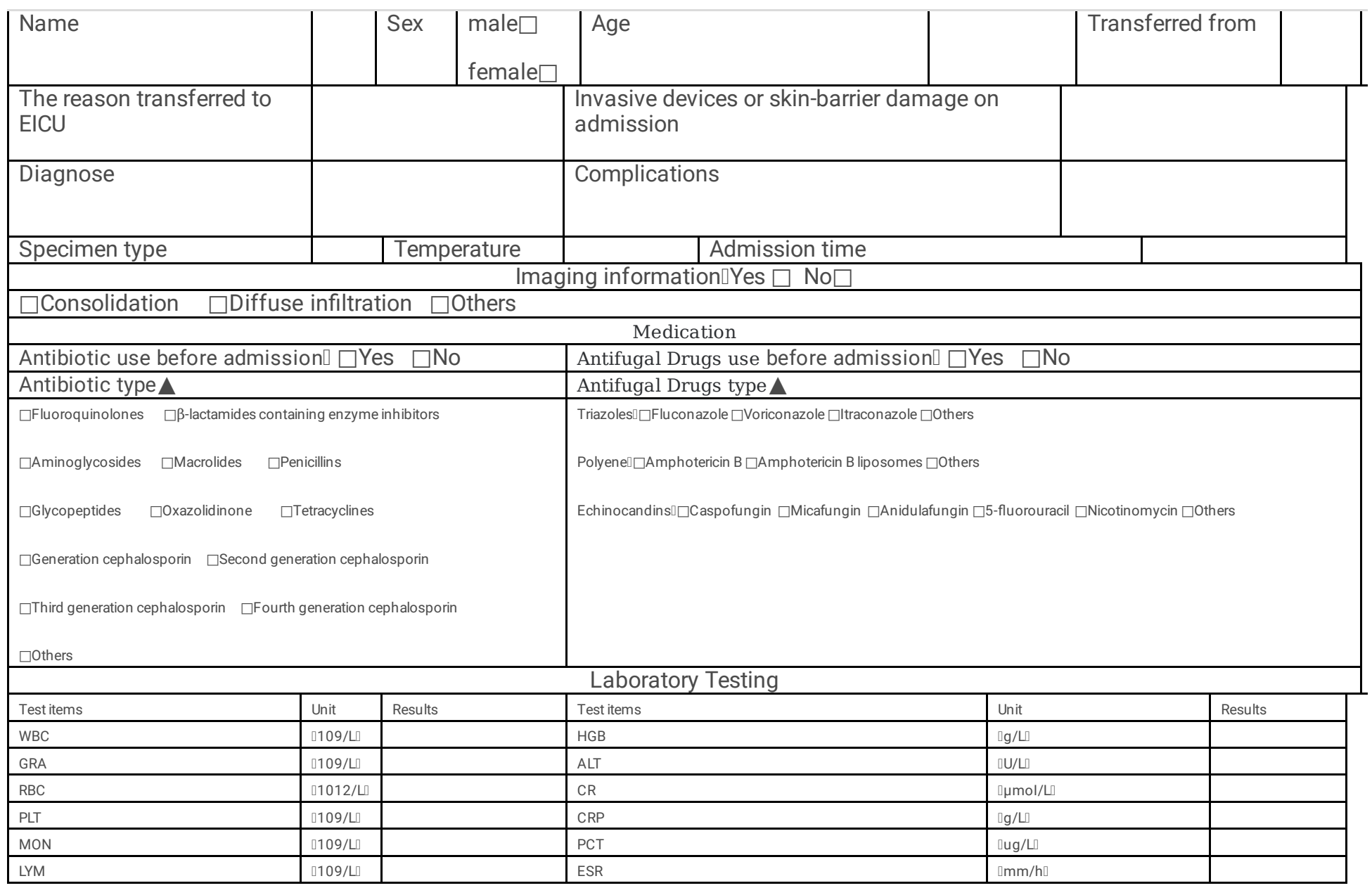

Stage 3 was conducted from February 2019 to April 2019, and focused on the efficiency and efficacy of the IPC interventions regarding prevention of nosocomial infections and whether the they could be carried out in clinical work without supervision [23]. Infection-control interventions undertaken to reduce the epidemic spread of CRE in 3 stages are listed in Fig 1. The clinical characteristics of the EICU patients were analysed once a month regarding their correlations with the Gene xpert results and mortality.

Fig 1 Infection-control interventions undertaken to reduce the epidemic spread of CRE in 3 stages. Other IPC interventions in stage 2 include multimodal strategy, hand hygiene, patient surveillance, contact precautions, patient isolation, environmental disinfection, environment surveillance, staff and living quarters monitoring and monitoring auditing and feedback..

\section{Active rapid molecular screening by GeneXpert Carba-R Assay}

A rectal swab was obtained from the patient by a paired-swab at admission to the EICU. One swab would be placed into the sample reagent vial and vortexed at high speed for 10 seconds. The prepared sample would be aspirated using the transfer pipette provided and transferred into the Xpert Carba-R Assay cartridge and then it would be put into the GeneXpert instrument. The results are given in 45 
minutes. Rapid active screenings by molecular detection were carried out immediately after the sampling by GeneXpert Carba-R Assay, simultaneously detecting five carbapenemase genes including blaKPC, blaIMP囚blaNDM囚blaVIM and blaOXA-48.

Bacterial isolation, antimicrobial susceptibility testing.

Surveillance cultures were sampled simultaneously with another unused rectal swab. Further routine surveillance cultures were obtained, including sputum, endotracheal aspirate, urinary tract, blood, and infection sites (individually selected according to patient's symptoms/differential diagnosis). The culture sampling has been repeated weekly or more often, when apposite (e.g. in case of fever, elevation of inflammatory markers etc.) Thus, we were able to monitor the incidence of CRE colonization or infection once a week or anytime if the patient got a fever or developed other symptoms of infections. All isolates were identified by the MALDI-TOF MS(Autof ms1000) and routine antibiotic susceptibility tests were performed by the VITEK2 compact system (bioMérieux, France) as to determine carbapenem resistance. The control strain was Escherichia coli ATCC 25922. Susceptibility breakpoints were interpreted as per the Clinical and Laboratory Standards Institute guidelines (CLSI M100-S28) [24].

\section{Multiplex PCR detect Carbapenemase genes}

Carbapenemase genes of the isolates collected from the rectal swabs culture of the patients on admission in EICU were verified by multiplex PCR with primers as shown below. (Table 2) [25]. In addition, a retrospective analysis of genetic correlations between the isolates from both rectal swabs on admission and the clinical culture during the hospitalization was performed by pulsed-field gel electrophoresis (PFGE). If the strains from different patients shared highly similar PFGE patterns, we suggested it might be a nosocomial infection.

Table 2 Oligonucleotides used in this study

\begin{tabular}{llll}
\hline Primer $^{\mathrm{a}}$ & Sequence $\left(5^{\prime}-3^{\prime}\right)$ & Gene & Product size $(\mathrm{bp})$ \\
\hline IMP-F & GGAATAGAGTGGCTTAAYTCTC & bla $_{I M P}$ & 232 \\
IMP-R & GGTTTAAYAAAACAACCACC & & \\
OXA-F & GCGTGGTTAAGGATGAACAC & bla $_{\text {OXA-48 }}$ & 438 \\
OXA-R & CATCAAGTTCAACCCAACCG & & \\
NDM-F & GGTTTGGCGATCTGGTTTTC & bla $_{\text {NDM }}$ & 621 \\
NDM-R & CGGAATGGCTCATCACGATC & & \\
KPC-Fm & CGTCTAGTTCTGCTGTCTTG & bla $_{K P C}$ & 798 \\
KPC-Rm & CTTGTCATCCTTGTTAGGCG & & 232 \\
VIM-F & GATGGTGTTTGGTCGCATA & bla & 390 \\
VIM-R & CGAATGCGCAGCACCAG & & \\
\hline
\end{tabular}

a F, sense primer; $R$, antisense primer.

\section{Statistical analysis}

All discontinuous variables (i.e. sex, invasive devices or skin-barrier damage on admission, antibiotic use before admission, outcomes) were compared between the xpert positive and negative groups using the $\chi 2$ test or Fisher's test. The mean of all continuous variables (i.e. age, WBC, CRP, PCT, days in hospital, and ICU days) was compared between the xpert positive and negative groups using MannWhitney U-test. The association between the xpert tests and the outcomes (alive or dead at discharge) active rapid molecular screening and IPC interventions were modeled by binary logistic regression. Two models were used: a crude model without any adjustment and a sex and age adjusted-model (continuous). All statistical analyses were performed using SPSS software, version 20.0. All tests were 2tailed, with $\mathrm{P}$ values $<0.05$ or a $95 \% \mathrm{Cl}$ excluding 1 were considered statistically significant.

\section{Results}

CRE nosocomial transmission present high prevalence in EICU. From the results from Gene Xpert detection, whether a patient carried carbapenemase genes on admission could be acquired in 1 hour. Isolation was conducted according to the result of molecular 
detection, even though culture results did not match exactly with it. (The phenotype and genotype of CRE did not match exactly.) A total of 217 patients were enrolled in the study. According to the results of Gene Xpert detection on admission, 23.0\% $\$ 50 / 217$ \patients carried the carbapenemase genes, $8.29 \%$ bla $_{\mathrm{KPC}} \otimes 12 / 217 \rrbracket, 9.68 \% \mathrm{bla}_{\mathrm{IMP}} \otimes 16 / 217 \rrbracket$ and $9.68 \% \varangle 15 / 217 \rrbracket \mathrm{bla}_{\mathrm{NDM}} \cdot 1.84 \% \varangle 4 / 217 \rrbracket$ patients carried a combination of 2 different carbapenemase genes and $1.38 \% \varangle 3 / 217 \rrbracket$ individuals carried a combination of 2 different carbapenemase genes 3 genes.( Fig 2) However, we did not get CRE isolation strains from all of the 50 positive sample although all GeneXpert findings were confirmed by culture, 18 isolates of CRE (16 isolates of CR-PK and 2 isolates of carbapenem-resistant Escherichia coli) from 16 patients were isolated by culture on MacConkey agar from the positive rectal swabs directly on admission. When the isolation strains were used to be verified by multiplex PCR, only one gene ( bla $_{\text {KPC }}$ or bla $a_{N D M}$ ) could be detected, even although Gene Xpert suggested presence of 3 different genes in one sample using the swab. The pulsed-field gel electrophoresis (PFGE) patterns were various among the isolates collected from 16 patients on admission. (Fig 3) The drug susceptibility information of 16 CRE strains isolated from rectal swab on admission and 14 CRE strains isolated from clinical culture from various specimens were showed in table 3.

Table 3 Antibiotic resistance characteristics of CRE strains

\begin{tabular}{|c|c|c|c|c|c|c|c|c|c|c|c|c|c|c|}
\hline & $\begin{array}{l}\text { Patient } \\
\text { ID }\end{array}$ & Gen & Imipenem & meropenem & Cefepime & Ceftriaxone & Cefazolin & Aztreonam & Amikacin & Ciprofloxacin & Gentamicin & Tobramycin & Tigecycline & $\begin{array}{l}\text { Piperacillin } \\
\text { plus } \\
\text { tazobactam }\end{array}$ \\
\hline $2018049 c^{\star}$ & 364827 & $\mathrm{KPC}$ & $>=16$ & 6 & 16 & $>=64$ & $>=64$ & $>=64$ & $<=2$ & $>=4$ & $>=16$ & $>=16$ & 20 & $>=128$ \\
\hline $2018083 c$ & 404003 & NDM & $>=16$ & 12 & $>=64$ & $>=64$ & $>=64$ & 8 & $<=2$ & $>=4$ & $>=16$ & 8 & 10 & $>=128$ \\
\hline $2018101 c$ & 318849 & KPC & $>=16$ & 6 & $>=64$ & $>=64$ & $>=64$ & $>=64$ & $<=2$ & $>=4$ & $<=1$ & $<=1$ & 20 & $>=128$ \\
\hline $2018144 c$ & 387313 & $\mathrm{KPC}$ & $>=16$ & 6 & $>=64$ & $>=64$ & $>=64$ & $>=64$ & $<=2$ & $>=4$ & $<=1$ & $<=1$ & 18 & $>=128$ \\
\hline $2018153 c$ & 420063 & $\mathrm{KPC}$ & $>=16$ & 6 & $>=64$ & $>=64$ & $>=64$ & $>=64$ & $<=2$ & $>=4$ & $<=1$ & $<=1$ & 19 & $>=128$ \\
\hline $2018160 c$ & 423231 & $\mathrm{KPC}$ & $>=16$ & 6 & $>=64$ & $>=64$ & $>=64$ & $>=64$ & $<=2$ & $>=4$ & $<=1$ & $<=1$ & 19 & $>=128$ \\
\hline $2018181 c$ & 427824 & $\mathrm{KPC}$ & $>=16$ & 6 & $>=64$ & $>=64$ & $>=64$ & $>=64$ & $>=64$ & $>=4$ & $>=16$ & $>=16$ & 18 & $>=128$ \\
\hline $2018186 c$ & 429027 & $\mathrm{KPC}$ & $>=16$ & 6 & $>=64$ & $>=64$ & $>=64$ & $>=64$ & $>=64$ & $>=4$ & $>=16$ & $>=16$ & 18 & $>=128$ \\
\hline $2018194 c$ & 430837 & $\mathrm{KPC}$ & $>=16$ & 6 & $>=64$ & $>=64$ & $>=64$ & $>=64$ & $<=2$ & $>=4$ & $<=1$ & $<=1$ & 18 & $>=128$ \\
\hline $2018199 c$ & 322466 & $\mathrm{KPC}$ & $>=16$ & 6 & $>=64$ & $>=64$ & $>=64$ & $>=64$ & $>=64$ & $>=4$ & $>=16$ & $>=16$ & 18 & $>=128$ \\
\hline $2018201 c$ & 427939 & $\mathrm{KPC}$ & $>=16$ & 6 & $>=64$ & $>=64$ & $>=64$ & $>=64$ & $>=64$ & $>=4$ & $>=16$ & $>=16$ & 22 & $>=128$ \\
\hline $2018211 c$ & 392880 & $\mathrm{KPC}$ & $>=16$ & 8 & $>=64$ & $>=64$ & $>=64$ & $>=64$ & $>=64$ & $>=4$ & $>=16$ & $>=16$ & 19 & $>=128$ \\
\hline 2018002 & 361673 & $\mathrm{KPC}$ & $>=16$ & 6 & $>=64$ & $>=64$ & $>=64$ & $>=64$ & $>=64$ & $>=4$ & $>=16$ & $>=16$ & 17 & $>=128$ \\
\hline 2018013 & 380030 & IPM $\square$ KPC & $>=16$ & 6 & $>=64$ & $>=64$ & $>=64$ & $>=16$ & $>=64$ & $>=4$ & $>=16$ & $>=16$ & 18 & $>=128$ \\
\hline 2018036 & 394953 & $\mathrm{KPC}$ & $>=16$ & 6 & $>=64$ & $>=64$ & $>=64$ & $>=64$ & $>=64$ & $>=4$ & $>=16$ & $>=16$ & 12 & $>=128$ \\
\hline 2018049 & 364827 & Kpc]IPM $\square$ NDM & $>=16$ & 6 & $>=64$ & $>=64$ & $>=64$ & $>=64$ & $>=64$ & $>=4$ & $>=16$ & $>=16$ & 19 & $>=128$ \\
\hline 2018101 & 318849 & $\mathrm{KPC}$ & $>=16$ & 6 & $>=64$ & $>=64$ & $>=64$ & $>=64$ & $<=2$ & $>=4$ & $<=1$ & $<=1$ & 21 & $>=128$ \\
\hline 2018109 & 405140 & 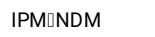 & $>=16$ & 12 & $>=64$ & $>=64$ & $>=64$ & $>=64$ & 4 & $>=4$ & 8 & $>=16$ & 12 & $>=128$ \\
\hline 2018131 & 353890 & $\mathrm{KPC}$ & 8 & 6 & 32 & $>=64$ & $>=64$ & $>=64$ & 8 & $>=4$ & $>=16$ & $>=16$ & 19 & $>=128$ \\
\hline 2018141 & 352267 & KPC & $>=16$ & 6 & $>=64$ & $>=64$ & $>=64$ & $>=64$ & $>=64$ & $>=4$ & $>=16$ & $>=16$ & 17 & $>=128$ \\
\hline 2018147 & 416772 & Kpc $I P M \square N D M$ & $>=16$ & 6 & $>=64$ & $>=64$ & $>=64$ & $>=64$ & $>=64$ & $>=4$ & $>=16$ & $>=16$ & 17 & $>=128$ \\
\hline 2018148 & 250464 & $\mathrm{Kpc} \rrbracket \mathrm{NDM}$ & $>=16$ & 6 & $>=64$ & $>=64$ & $>=64$ & 4 & $>=64$ & $>=4$ & $>=16$ & $>=16$ & 23 & 64 \\
\hline 2018152 & 318849 & $\mathrm{KPC}$ & $>=16$ & 6 & $>=64$ & $>=64$ & $>=64$ & $>=64$ & $<=2$ & $>=4$ & $<=1$ & $<=1$ & 19 & $>=128$ \\
\hline 2018157 & 418786 & $\mathrm{KPC}$ & $>=16$ & 6 & $>=64$ & $>=64$ & $>=64$ & $>=16$ & $<=2$ & $>=4$ & $>=16$ & $<=1$ & 14 & $>=128$ \\
\hline 2018190 & 378142 & Kpc $I P M \square N D M$ & $>=16$ & 6 & $>=64$ & $>=64$ & $>=64$ & $>=64$ & $<=2$ & $>=4$ & $<=1$ & $<=1$ & 18 & $>=128$ \\
\hline 2018201 & 427939 & $\mathrm{KPC}$ & $>=16$ & 6 & $>=64$ & $>=64$ & $>=64$ & $>=64$ & $>=64$ & $>=4$ & $>=16$ & $>=16$ & 18 & $>=128$ \\
\hline 2018204 & 381155 & NDM & $>=16$ & 6 & $>=64$ & $>=64$ & $>=64$ & $>=64$ & 16 & $>=4$ & $>=16$ & $>=16$ & 22 & $>=128$ \\
\hline
\end{tabular}

c. Stains collected from clinical culture during the hospitalization.

Fig 2 The distribution of 50 positive samples confirmed by Gene Xpert detection. Most patients (43/50) carried individual carbapenemase gene and $b / a_{K P C}, b / a_{I M P}$ and $b / a_{N D M}$ account for $24 \%, 32 \%$ and $30 \%$ respectively.

a.KPC represents $b / a_{K P C}, I M P$ represents $b / a_{I M P}$ and NDM represents $b / a_{N D M}$ in Fig 2

Fig 3 The dendrogram of PFGE results of CRE strains. The PFGE patterns were various among the isolates collected from 16 patients. 
The clinicopathological characteristics of the included patients, laboratory and Gene Xpert test results, duration of the hospitalization and outcomes were recorded in Table 4. The mean age of the patients suggests the elder patients were more likely to carry Carbapenemase genes $(p=0.006)$. Patients with invasive devices or skin-barrier damage on admission $(p=0.017)$ and those who used antibiotics before admission $(p<0.001)$ were more likely to be colonized or infected by CRE, as shown in Fig 4 . The latter observation confirms that an overuse of antibiotics increases the risk of CRE infections. There were no significant association between the xpert test and the outcome, in sex and gender-adjusted cohort $(p=0.114)$. The details of clinicopatholigical/laboratory characteristics are showed in additional file 1.

Table 4 Clinicopatholigical/laboratory characteristics and outcomes

\begin{tabular}{|c|c|c|c|}
\hline Number(\%) & xpert+ & xpert- & $P$ value \\
\hline sex & & & 0.889 \\
\hline male & $29 \llbracket 58 \rrbracket$ & $95 \llbracket 57 \rrbracket$ & \\
\hline female & $21 \rrbracket 42 \rrbracket$ & $72 \varangle 43 \rrbracket$ & \\
\hline age & & & $0.006^{*}$ \\
\hline mean $\pm s d$ & $78.8 \pm 9.5$ & $72.3 \pm 15.5$ & \\
\hline median (interquartile ) & $81.5(70-86)$ & $75(65-84)$ & \\
\hline Invasive devices or skin-barrier damage on admission & & & $0.017^{\star}$ \\
\hline YES & $5(10)$ & $3(2)$ & \\
\hline NO & $45(90)$ & $164(98)$ & \\
\hline antibiotic use before admission & & & $<0.001^{*}$ \\
\hline YES & $15(30)$ & $33(20)$ & \\
\hline NO & $35(70)$ & $134(80)$ & \\
\hline WBC 0 & & & 0.485 \\
\hline mean $\pm s d$ & $11.8 \pm 8.2$ & $11.1 \pm 5.8$ & \\
\hline CRP (number $=216$ ) & & & 0.404 \\
\hline mean $\pm s d$ & $43 \pm 55$ & $110 \pm 563$ & \\
\hline median (interquartile) & $30(2-50)$ & $41(10-100)$ & \\
\hline PCT (number= 215 ) & & & 0.54 \\
\hline mean $\pm s d$ & $6.93 \pm 14.83$ & $5.4 \pm 15.44$ & \\
\hline median (interquartile ) & $0.67(0.21-5.63)$ & $0.33(0.11-2.28)$ & \\
\hline Inhospital days & & & 0.49 \\
\hline median (interquartile range) & $12.5(9-17)$ & $12(8-18)$ & \\
\hline EICU stay (days) & & & 0.934 \\
\hline median (interquartile range) & $9.5(6-15)$ & $8(4-14)$ & \\
\hline outcome & & & 0.114 \\
\hline improvement & $32(64)$ & 106(64) & \\
\hline discharge on voluntary & $1(2)$ & $18(10)$ & \\
\hline death & $17(34)$ & $43(26)$ & \\
\hline
\end{tabular}

Clinicopatholigical/laboratory characteristics and outcomes of CRE colonized/infected and not colonized/infected EICU patients, as per Gene Xpert result.

Fig 4 Association between antibiotics/skin damage and the result of Gene Xpert test. Antibiotic $=$ the patient used antibiotics before admission. Wound $=$ the patient had invasive devices or skin-barrier damage on admission. Patients who used antibiotics prior to admission, had invasive devices or skin damage tended to have Gene Xpert positive test result (infected or colonized with CRE).

IPC interventions effectively reduce the colonization and infection of CRE in EICU through active rapid molecular screening. The baseline rate of CRE colonization or infection rate identified by routine clinical culture detection decreased from $3.37 \%$ significantly to $1.20 \%(p=$ 
0.0438) during the main experimental stage (stage 2), supporting the primary hypothesis that rapid active screening by molecular detection in combination with other IPC intervention reduces nosocomial CRE infection. However, we observed a raise of the rate increased to $6.12 \%$ in stage 3 (Table 5), suggesting that rapid active screening by molecular detection and supervised IPC interventions lead to an effective decrease in CRE infection, while routine checks and unsupervised interventions fail to maintain this effect. In order to exclude effect of any change in in-hospital patients in stage 2 and stage 3 . The main diagnoses in both stages were listed in table 6 from most frequent to least frequent. There was no significant difference in diseases between stage 2 and stage 3 . We compared the duration of total in-hospital and in-EICU stay in stage 2 and stage 3. (Fig 5) It showed no significant difference both in total in-hospital and in-EICU stay between stage 2 and stage 3 which meant the increase of detection rate might not be caused by a longer duration.

Table 5 Detection rates of CRE colonization/ infection in EICU

\begin{tabular}{llllll}
\hline Intervention stage & No. of patients & No. of cases & The detection rates of CRE & $\mathrm{P}^{\mathrm{a}}$ & $\mathrm{P}^{\mathrm{b}}$ \\
\hline Baseline period & 168 & 15 & $3.37 \%(15 / 445)$ & $/$ & $/$ \\
Stage 2 & 106 & 5 & $1.20 \%(5 / 418)$ & $0.0438^{\star}$ & $/$ \\
Stage 3 & 46 & 9 & $6.12 \%(9 / 147)$ & 0.0812 & $0.0010^{*}$ \\
\hline
\end{tabular}

${ }^{\mathrm{a}}$ Compared with baseline

${ }^{\mathrm{b}}$ Compared with stage 2

Table 6 Diagnoses Infectious Complications and comorbidities of the patients in EICU.

\begin{tabular}{lll}
\hline Diagnose & Infectious Complication & Comorbidity \\
\hline Pulmonary infection & Acute exacerbation of chronic bronchitis & Hypertension \\
Sepsis & Respiratory failure & Diabetes \\
Gastrointestinal hemorrhage & Bronchial asthma & Anemia \\
Cerebral infarction & Severe pneumonia & Electrolyte disturbance \\
Acute heart failure & Pleural effusion & Hypoproteinemia \\
Uremia & Aspiration pneumonia & Coronary heart disease \\
Acute pancreatitis & Interstitial pneumonia & Hypohepatia \\
$\ldots$ & Chronic obstructive pulmonary disease acute episode & $\ldots$ \\
\hline
\end{tabular}

Fig 5 The duration of total inhospital and inEICU stay in stage 2 and stage 3 . The duration showed no significant difference both in total inhospital and inEICU stay between stage 2 and stage 3.

\section{Discussion}

Carbapenem antibiotics are extensively used worldwide due to their strong antibacterial activity and broad spectrum [26]. CRE infections are increasingly challenging due to a growing antimicrobial resistance and thus causative for a high mortality. [27] [28] In this study, we conducted a series of planned and protocolized IPC interventions in order to establish if the combination of these interventions and measures of detection and monitoring of CRE colonizations/infections (active screen at admission) by a rapid, semi-nested real-time fluorescent PCR, is effective in reduction of infections, mortality and morbidity of EICU patients.

The PFGE result suggests that it was not a nosocomial infection outbreak since the patients were not infected with the same clone of the pathogen. (Fig 3) The sample collected from the patients on admission and the clinical culture during their stay in EICU showed similar PFGE patterns. We compared the PFGE to various patterns, such as bed in the ward, time of admission, the collection time of the samples and where the patients were transferred from. Except for the strains collected form the same patient, almost no 
intercorrelations between the patients were found between for their PFGEs and clinical characteristics. Solely the common strains 2018208 and 2018211 were identified in various cultures, both with a very similar PFGE pattern, which strongly suggests a nosocomial infection.

Increased participation during the education phase of the intervention program was reported as an important factor to decrease the incidence of carbapenem-resistant pathogen infections. [29]. Feedback from screening services, rapid turnaround time and efficient communication were all positively correlated to overall success in outbreak control[29]. Based on this study, we designed and designated one entire month for educating and training of medical professionals and health care workers, familiarizing them with the study-specific IPC intervention model, screening measures, general hygiene. These educational approaches resulted in higher compliance and better adherence to study protocols, as well as in further reduction of the infection rates.

The existing evidence regarding the effectiveness of isolation and precautions in controlling spread of CRE is inconsistent. A 4-year perspective study in Israel showed that isolation precautions alone are ineffective in IPC [30]. A similar 4-year quasi-experimental study in China suggested that bundled IPC interventions, including isolation precaution, rapidly decrease the incidence of ICU-acquired CR-KP colonisations/infections. [24] Due to limited space and large number of patients in the EICU ward, the number of isolation rooms is often limited, just as in our institution. Not all CRE-positive patients at the time of admission can be placed in a separate isolation, so a clinical isolation as allowed by factual conditions is offered. Our results confirm the hypothesis that combined IPC and isolation precautions are most effective, especially in the real-world conditions with kimited ressources.

Our findings suggest that environmental surveillance is of high importance in IPC interventions. The "environment" is defined as all areas of the ward, involved hand colonization and hygiene, clothing of medical staff and medical staff themselves. [31] Environmental monitoring was helpful in providing a clear feedback to health care providers about disinfection processes. In our study, environmental monitoring was conducted twice a month. If case of unsatisfactory outcomes, the ward underwent a total disinfection process with an additional surveillance thereafter. Such approach was efficient and effective in CRE reduction and positive in terms of individual hygiene awareness of the health care providers.

In the third phase of the study, the incidence of CRE increased to $6.12 \%$, which was almost twice of the baseline value. We could exclude patient-related factors as causative for such observation, since in the second stage, the positive rate of patient Xpert admission was $24.20 \%$ (38/157), while the positive rate of Xpert gene in the third stage was only $20 \%(12 / 60)$. Further investigation found that employees were not strictly following IPC interventions when there was no supervision (stage 3 ). In addition, during the third phase, there was a temporary absence of the head nurse, while the replacement was not trained accordingly in the pre-phase of our study (educational month). This highlights the importance of continuous education and specific training for the intervention processes.

In addition to our main findings, our study allowed to investigate Gene Xpert as a molecular detection method, previously developed to help antibiotic selection at diagnosis of CRE infection, allowing for a rapid assay and active screening that detects drug resistance genes and facilitates the clinical selection of antibiotics at the time point of CRE infection diagnosis. [32] This molecular diagnostic testing technology reports results within 1 hour, allowing clinicians to diagnose CRE infections in a timely manner and to undertake appropriate treatment and management measures and isolate patients to avoid further spread of bacteria. [33]

There are some limitations to our study. Firstly, it was a single-center study, with a limited number of participating patients. Secondly, due to large discrepancies in different health care systems, the clinical relevance of the result may be limited to hospitals in Asia/China. Thirdly, the statistical significance of the relation between the clinical characteristics and patients' outcomes was not established, which could be attributed to a small sample size rather than to an actual lack of correlations. The assessment of how strictly the IPC interventions were conducted was mainly subjective.

\section{Conclusions}

We are the first to report objective data on how IPC interventions with active rapid molecular screening appropriately implemented in a Chinese hospital can contribute to an actual reduction of CRE infection rate. Our protocols can be used as a base for practical real-world recommendations, implementable in Chinese hospitals. Further prospective studies in a multicenter settings are warranted in order to provide valid clinical research data for the development of multi-drug resistance prevention and control guidelines for China.

\section{Abbreviations}


IPC Infection-prevention and control

CRE Carbapenem-resistant Enterobacteriaceae

EICU Emergency intensive care unit

PCR Polymerase chain reaction

CDC Centers for Disease Control

ESBL Extended Spectrum Beta-Lactamase

CR-GNB Carbapenem-resistant gram-negative bacilli

CRAB Carbapenem-resistant Acinetobacter baumannii

CRPsA Carbapenem-resistant Pseudomonas aeruginosa

CR-KP Carbapenem-resistant Klebsiella pneumoniae

CRF Case Report Form

PFGE Pulsed-field gel electrophoresis

\section{Declarations}

Ethics approval and consent to participate

Ethical approval was obtained from Shanghai East Hospital(Tongji University School of Medicine) in April 2018. The approval No.is $\varangle 2018 \rrbracket(07)$. All study participants and/or their immediate guardians, signed a detailed informed consent form prior to the study enrollment. The Ethical approval file is provided in additional file 2.

\section{Consent for publication}

Not applicable

\section{Availability of data and materials}

All data analysed during this study are provided in the attached file(Additional file 1).

\section{Competing interests}

All authors declare that there are no competing interests.

\section{Funding}

The study was sponsored by the Clinical Peak Discipline/Clinical Plateau Discipline『PWYgf2018-05区, Shanghai Key Medical Discipline for Critical Care Medicine 『No. 2017ZZ02017区, Municipal Human Resources Development Program for Outstanding Leaders in Medical Disciplines in Shanghai (grant number 2017BR032).

\section{Acknowledgements}

Not applicable.

Authors' information

Simin Yang, Lihua He and Ke Li contributed equally to this work.

Affiliations

Simin Yang, Xiaoyu Yu, Lijun Ni, Liang Hu, Jian Guo and Wenjuan Wu 
Department of Laboratory Medicine, Shanghai East Hospital, Tongji University School of Medicine, Shanghai, China

Ke Li and Lunxian Tang

Department of Hospital Infection Management, Shanghai East Ho spital, Tongji University School of Medicine, Shanghai, China

Lihua He

Department of Emergency Intensive Care Unit, Shanghai East Hospital, Tongji University School of Medicine, Shanghai, China

Ewelina Biskup

Shanghai University of Medicine and Health Sciences, Shanghai, PRC//University Hospital of Basel, Department of Internal Medicine, Petersgraben 4, 4051 Basel, Switzerland

Authors' contributions

Simin Yang and Wenjuan Wu contributed to the study or experimental design. Lijun Ni, Liang Hu, Jian Guo, Lihua He and Ke Li conducted the experiments. Simin Yang and Xiaoyu Yu analyzed the data. Simin Yang and Lunxian Tang organized the clinical trial design and its operation. Simin Yang and Ewelina Biskup wrote the manuscript. All authors contributed to writing, editing, and approving the manuscript.

Corresponding author

Lunxian Tang, 456tlx@163.com Wenjuan Wu,wwj1210@126.com

\section{References}

1.Carbapenem-resistant Enterobacteriaceae: a growing threat. Nursing 2013, 43(7):65.

2.Zhanel GG, Lawrence CK, Adam H, Schweizer F, Zelenitsky S, Zhanel M, Lagace-Wiens PRS, Walkty A, Denisuik A, Golden A et al: Imipenem-Relebactam and Meropenem-Vaborbactam: Two Novel Carbapenem-beta-Lactamase Inhibitor Combinations. Drugs 2018, 78(1):65-98.

3.Solomon SL, Oliver KB: Antibiotic resistance threats in the United States: stepping back from the brink. American family physician 2014, 89(12):938-941.

4.Zhang R, Liu L, Zhou H, Chan EW, Li J, Fang Y, Li Y, Liao K, Chen S: Nationwide Surveillance of Clinical Carbapenem-resistant Enterobacteriaceae (CRE) Strains in China. EBioMedicine 2017, 19:98-106.

5.Rumbo C, Gato E, Lopez M, Ruiz de Alegria C, Fernandez-Cuenca F, Martinez-Martinez L, Vila J, Pachon J, Cisneros JM, RodriguezBano J et al: Contribution of efflux pumps, porins, and beta-lactamases to multidrug resistance in clinical isolates of Acinetobacter baumannii. Antimicrobial agents and chemotherapy 2013, 57(11):5247-5257.

6.Kim YA, Park YS: Epidemiology and treatment of antimicrobialresistant gram-negative bacteria in Korea. The Korean journal of internal medicine 2018, 33(2):247-255.

7.Martens E, Demain AL: The antibiotic resistance crisis, with a focus on the United States. The Journal of antibiotics 2017, 70(5):520526.

8.Canton R, Akova M, Carmeli Y, Giske CG, Glupczynski Y, Gniadkowski M, Livermore DM, Miriagou V, Naas T, Rossolini GM et al: Rapid evolution and spread of carbapenemases among Enterobacteriaceae in Europe. Clinical microbiology and infection: the official publication of the European Society of Clinical Microbiology and Infectious Diseases 2012, 18(5):413-431.

9.McConville TH, Sullivan SB, Gomez-Simmonds A, Whittier S, Uhlemann AC: Carbapenem-resistant Enterobacteriaceae colonization (CRE) and subsequent risk of infection and 90-day mortality in critically ill patients, an observational study. PloS one 2017, 12(10):e0186195. 
10.Bartsch SM, McKinnell JA, Mueller LE, Miller LG, Gohil SK, Huang SS, Lee BY: Potential economic burden of carbapenem-resistant Enterobacteriaceae (CRE) in the United States. Clinical microbiology and infection: the official publication of the European Society of Clinical Microbiology and Infectious Diseases 2017, 23(1):48 e49-48 e16.

11.Bassetti M, Poulakou G, Ruppe E, Bouza E, Van Hal SJ, Brink A: Antimicrobial resistance in the next 30 years, humankind, bugs and drugs: a visionary approach. Intensive care medicine 2017, 43(10):1464-1475.

12.Durante-Mangoni E, Andini R, Zampino R: Management of carbapenem-resistant Enterobacteriaceae infections. Clinical microbiology and infection: the official publication of the European Society of Clinical Microbiology and Infectious Diseases 2019, 25(8):943-950.

13.Chinese XDRCWG, Guan X, He L, Hu B, Hu J, Huang X, Lai G, Li Y, Liu Y, Ni Y et al: Laboratory diagnosis, clinical management and infection control of the infections caused by extensively drug-resistant Gram-negative bacilli: a Chinese consensus statement. Clinical microbiology and infection: the official publication of the European Society of Clinical Microbiology and Infectious Diseases 2016,22 Suppl 1:S15-25.

14.Hu FP, Guo Y, Zhu DM, Wang F, Jiang XF, Xu YC, Zhang XJ, Zhang CX, Ji P, Xie Y et al: Resistance trends among clinical isolates in China reported from CHINET surveillance of bacterial resistance, 2005-2014. Clinical microbiology and infection: the official publication of the European Society of Clinical Microbiology and Infectious Diseases 2016, 22 Suppl 1:S9-14.

15.Friedman ND, Carmeli Y, Walton AL, Schwaber MJ: Carbapenem-Resistant Enterobacteriaceae: A Strategic Roadmap for Infection Control. Infection control and hospital epidemiology 2017, 38(5):580-594.

16.. In: Guidelines for the Prevention and Control of Carbapenem-Resistant Enterobacteriaceae, Acinetobacter baumannii and Pseudomonas aeruginosa in Health Care Facilities. edn. Geneva; 2017.

17.Bar-Yoseph H, Hussein K, Braun E, Paul M: Natural history and decolonization strategies for ESBL/carbapenem-resistant Enterobacteriaceae carriage: systematic review and meta-analysis. J Antimicrob Chemother 2016, 71(10):2729-2739.

18.Ramanathan YV, Venkatasubramanian R, Nambi PS, Ramabathiran M, Venkataraman R, Thirunarayan MA, Samundeeswari P, Ramakrishnan N: Carbapenem-resistant enterobacteriaceae screening: A core infection control measure for critical care unit in India? Indian journal of medical microbiology 2018, 36(4):572-576.

19.Kurtz SL: Introduction of New Theory for Hand Hygiene Surveillance: Healthcare Environment Theory. Res Theory Nurs Pract 2018, 32(2):144-167.

20.Martischang R, Buetti N, Balmelli C, Saam M, Widmer A, Harbarth S: Nation-wide survey of screening practices to detect carriers of multi-drug resistant organisms upon admission to Swiss healthcare institutions. Antimicrobial resistance and infection control 2019 , 8:37.

21.Mathers AJ, Vegesana K, German Mesner I, Barry KE, Pannone A, Baumann J, Crook DW, Stoesser N, Kotay S, Carroll J et al: Intensive Care Unit Wastewater Interventions to Prevent Transmission of Multispecies Klebsiella pneumoniae Carbapenemase-Producing Organisms. Clinical infectious diseases: an official publication of the Infectious Diseases Society of America 2018, 67(2):171-178.

22.Suen LKP, Siu GKH, Guo YP, Yeung SKW, Lo KYK, O’Donoghue M: The public washroom - friend or foe? An observational study of washroom cleanliness combined with microbiological investigation of hand hygiene facilities. Antimicrobial resistance and infection control 2019, 8:47.

23.Wang J, Liu F, Tan JBX, Harbarth S, Pittet D, Zingg W: Implementation of infection prevention and control in acute care hospitals in Mainland China - a systematic review. Antimicrobial resistance and infection control 2019, 8:32.

24.Li M, Wang X, Wang J, Tan R, Sun J, Li L, Huang J, Wu J, Gu Q, Zhao Y et al: Infection-prevention and control interventions to reduce colonisation and infection of intensive care unit-acquired carbapenem-resistant Klebsiella pneumoniae: a 4-year quasi-experimental before-and-after study. Antimicrobial resistance and infection control 2019, 8:8.

25.Poirel L, Walsh TR, Cuvillier V, Nordmann P: Multiplex PCR for detection of acquired carbapenemase genes. Diagn Microbiol Infect Dis 2011, 70(1):119-123.

Page 12/16 
26.Cerceo E, Deitelzweig SB, Sherman BM, Amin AN: Multidrug-Resistant Gram-Negative Bacterial Infections in the Hospital Setting: Overview, Implications for Clinical Practice, and Emerging Treatment Options. Microb Drug Resist 2016, 22(5):412-431.

27.Alvi N, Tipoo FA, Imran A, Ashraf MN, Qidwai A, Khursheed M, Moiz B, Adil SN, Fadoo Z, Altaf S et al: Burden of Cardiac Siderosis in a Thalassemia-Major Endemic Population: A Preliminary Report From Pakistan. J Pediatr Hematol Oncol 2016, 38(5):378-383.

28.Allegranzi B, Bagheri Nejad S, Combescure C, Graafmans W, Attar H, Donaldson L, Pittet D: Burden of endemic health-care-associated infection in developing countries: systematic review and meta-analysis. Lancet 2011, 377(9761):228-241.

29.Kousouli E, Zarkotou O, Politi L, Polimeri K, Vrioni G, Themeli-Digalaki K, Tsakris A, Pournaras S: Infection control interventions affected by resource shortages: impact on the incidence of bacteremias caused by carbapenem-resistant pathogens. Eur J Clin Microbiol Infect Dis 2018, 37(1):43-50.

30.Cohen MJ, Block C, Levin PD, Schwartz C, Gross I, Weiss Y, Moses AE, Benenson S: Institutional control measures to curtail the epidemic spread of carbapenem-resistant Klebsiella pneumoniae: a 4-year perspective. Infection control and hospital epidemiology 2011, 32(7):673-678.

31.Pfrimmer DM, Johnson MR, Guthmiller ML, Lehman JL, Ernste VK, Rhudy LM: Surveillance: A Nursing Intervention for Improving Patient Safety in Critical Care Environment. Dimens Crit Care Nurs 2017, 36(1):45-52.

32.Skodvin B, Wathne JS, Lindemann PC, Harthug S, Nilsen RM, Charani E, Syre H, Kittang BR, Kleppe LKS, Smith I: Use of microbiology tests in the era of increasing AMR rates- a multicentre hospital cohort study. Antimicrobial resistance and infection control $2019,8.28$.

33.David S, Reuter S, Harris SR, Glasner C, Feltwell T, Argimon S, Abudahab K, Goater R, Giani T, Errico G et al: Epidemic of carbapenemresistant Klebsiella pneumoniae in Europe is driven by nosocomial spread. Nature microbiology 2019.

\section{Figures}

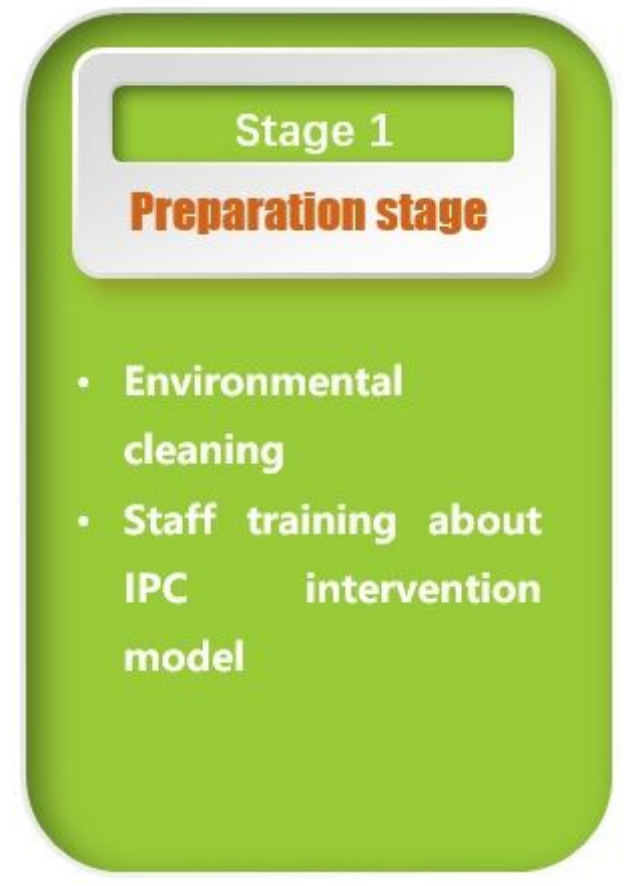

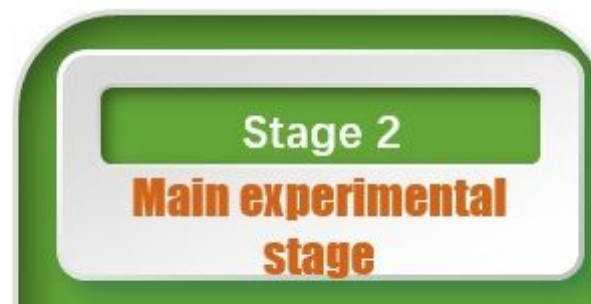

- Rapid active screening by molecular detection

- Other IPC interventions

- Multimodal strategy

- Sink monitoring

- Avoiding dust

- Routine clinical culture

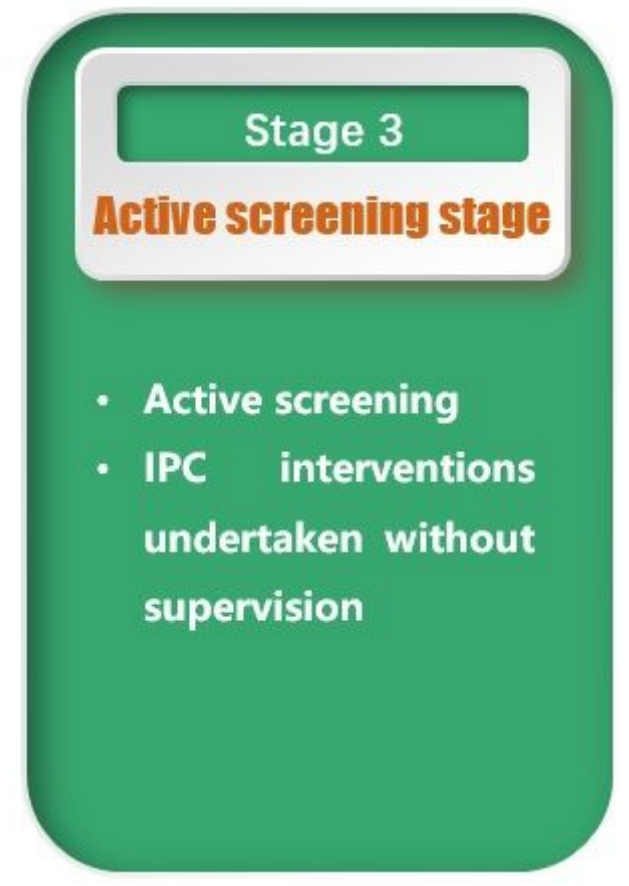

\section{Figure 1}

Infection-control interventions undertaken to reduce the epidemic spread of CRE in 3 stages. Other IPC interventions in stage 2 include multimodal strategy, hand hygiene, patient surveillance, contact precautions, patient isolation, environmental disinfection, environment surveillance, staff and living quarters monitoring and monitoring auditing and feedback. 


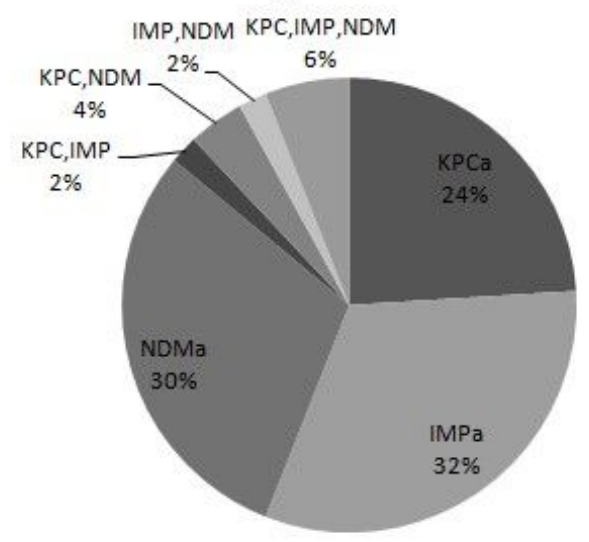

\section{Figure 2}

The distribution of 50 positive samples confirmed by Gene Xpert detection. Most patients (43/50) carried individual carbapenemase gene and blaKPC, blaIMP and blaNDM account for $24 \%, 32 \%$ and $30 \%$ respectively. a.KPC represents blaKPC, IMP represents blaIMP and NDM represents blaNDM in Fig 2

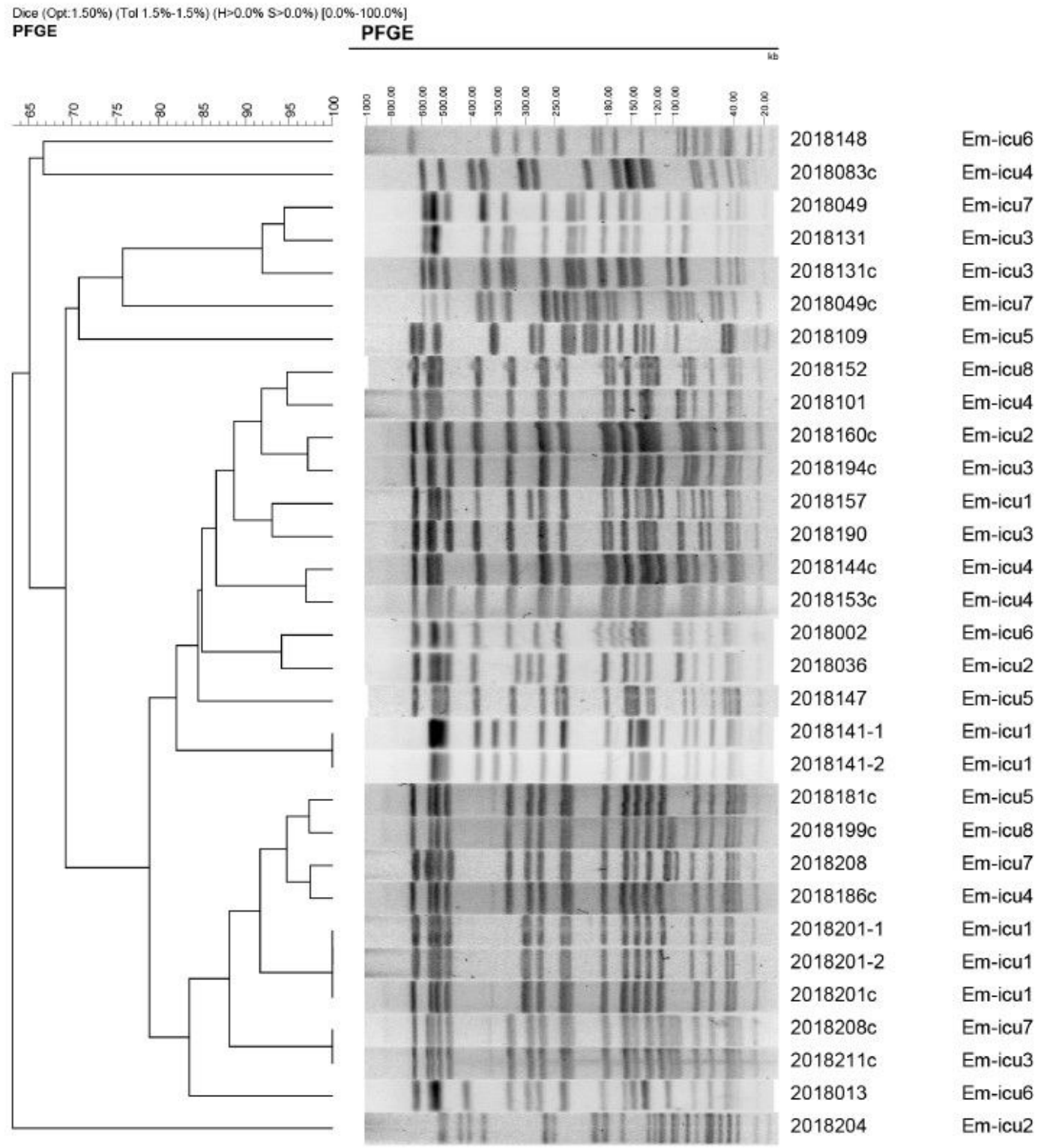




\section{Figure 3}

The dendrogram of PFGE results of CRE strains. The PFGE patterns were various among the isolates collected from 16 patients.

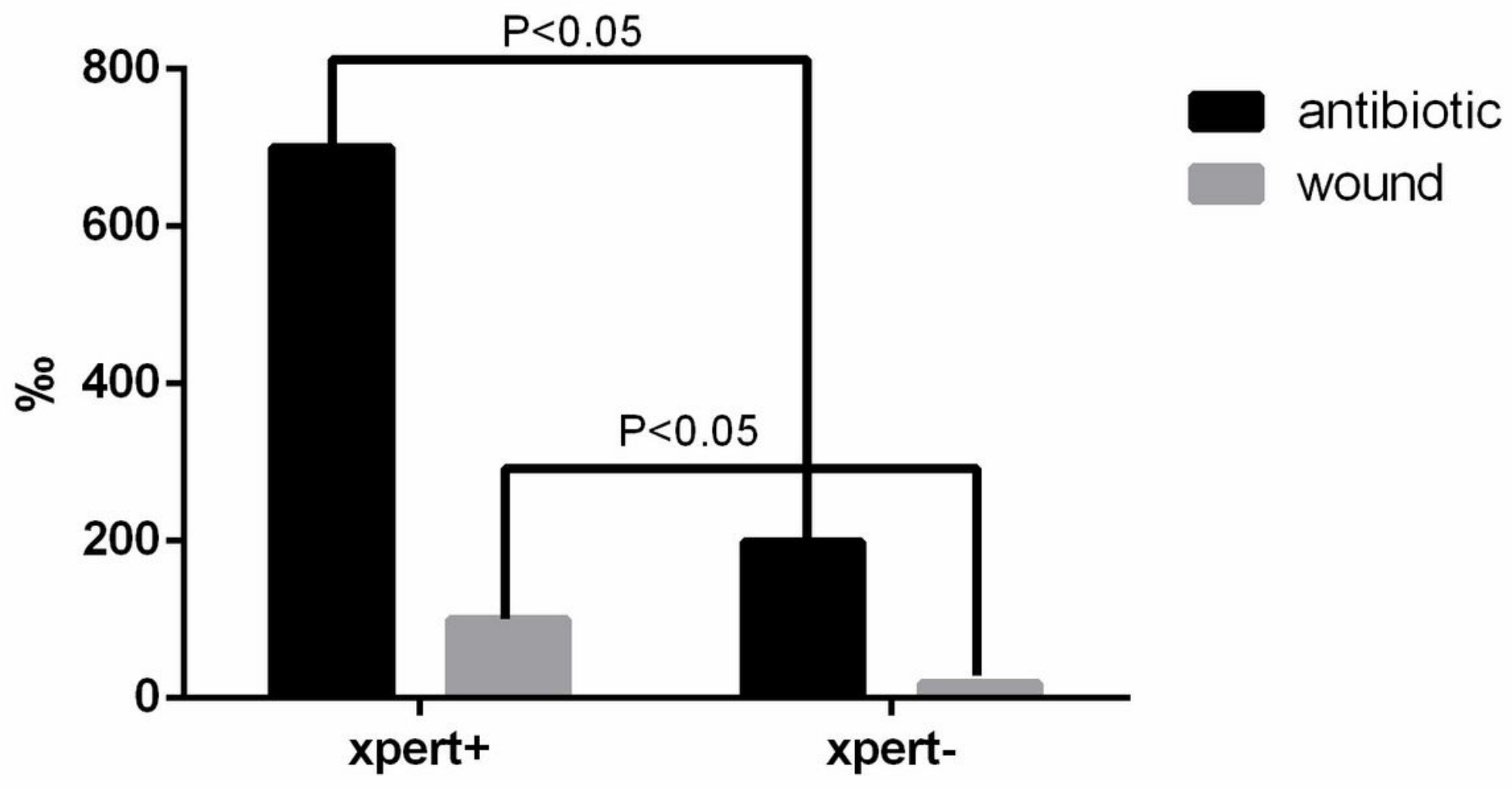

Figure 4

Association between antibiotics/skin damage and the result of Gene Xpert test. Antibiotic = the patient used antibiotics before admission. Wound $=$ the patient had invasive devices or skin-barrier damage on admission. Patients who used antibiotics prior to admission, had invasive devices or skin damage tended to have Gene Xpert positive test result (infected or colonized with CRE). 


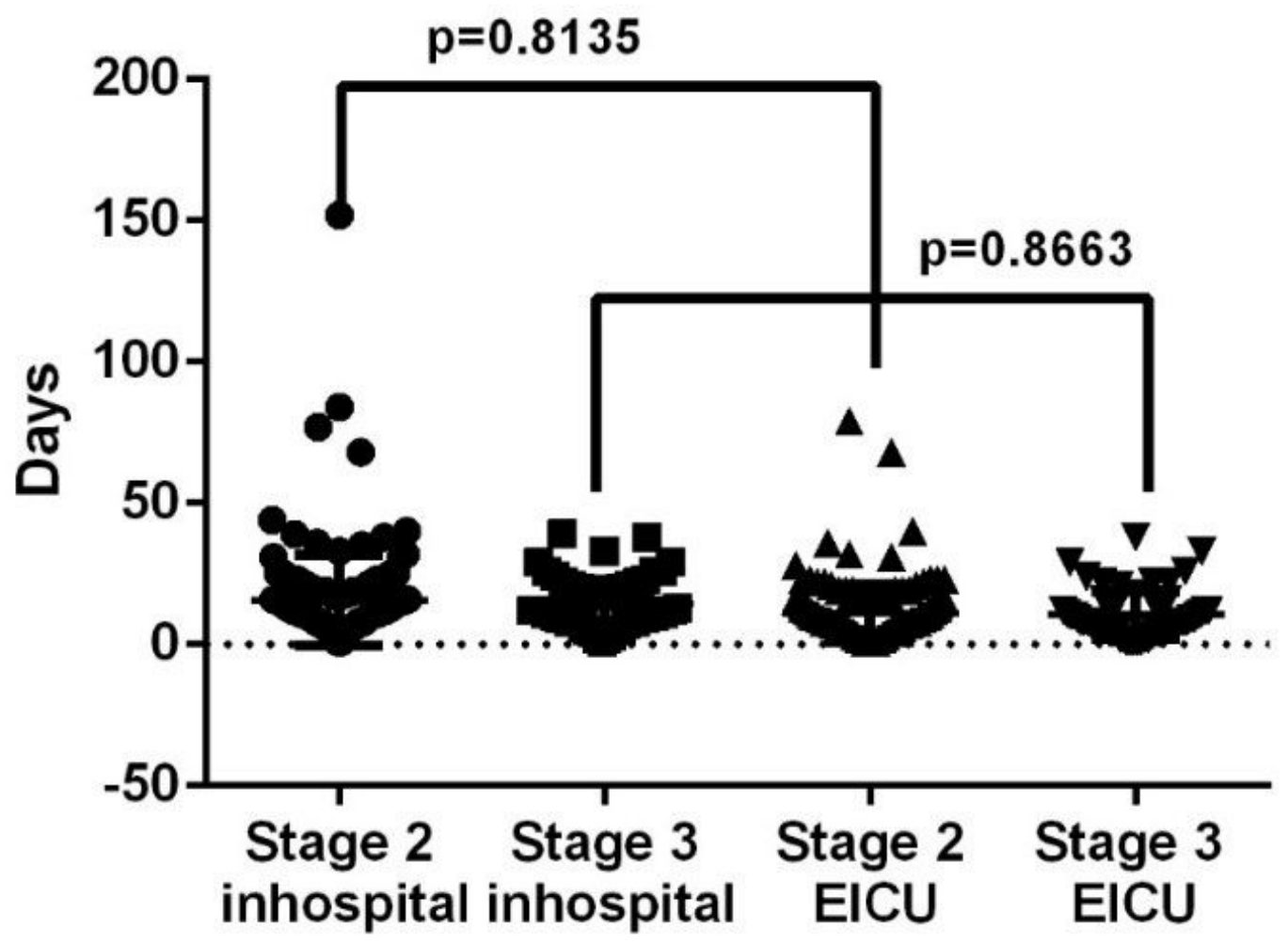

Figure 5

The duration of total inhospital and inEICU stay in stage 2 and stage 3 . The duration showed no significant difference both in total inhospital and inEICU stay between stage 2 and stage 3 .

\section{Supplementary Files}

This is a list of supplementary files associated with this preprint. Click to download.

- Additionalfile1.xls

- Additionalfile2.docx 